\title{
What is spinodal decomposition?
}

\author{
E. P. Favvas ${ }^{*}, 1$ and A. Ch. Mitropoulos ${ }^{2}$ \\ ${ }^{1}$ Institute of Physical Chemistry, NCSR "Demokritos", 15310 Ag.Paraskevi, Attikis, Greece. \\ ${ }^{2}$ Department of Petroleum Technology, Kavala Institute of Technology, 65404 St.Lucas, Kavala, Greece.
}

Received 17 January 2008; Accepted 20 February 2008

\begin{abstract}
Abstract: Phase separation may occur in a way that the growth is not in extent but in amplitude. Only in the unstable region such a procedure is thermodynamically feasible. In a phase diagram the unstable region is defined by the spinodal. When a system has crossed this locus, phase separation occurs spontaneously without the presence of a nucleation step. This process is known as spinodal decomposition and commonly results to a high interconnectivity of the two phases. The Cahn-Hilliard equation describes the kinetics of the process. In this note both processes (nucleation and spinodal) are depicted schematically.
\end{abstract}

Keywords: spinodal decomposition, nucleation and growth.

A pair of partially miscible liquids, i.e. liquids that do not mix in all proportions at all temperatures, shows in a temperature-composition diagram a miscibility gap where phase separation occurs. Gibbs [1] showed that the condition for stability (or metastability) in respect to continuous change of phase is that the second derivative of the free energy of mixing to be positive. If negative, the system is unstable. If zero, the spinodal is defined. The free energy of mixing, $\Delta \mathrm{G}^{\mathrm{mix}}$, has the following general form:

$$
\Delta G^{m i x}=\Delta H^{m i x}-T \Delta S^{m i x}
$$

For the regular solution model [2] the entropy of mixing is the same as for the ideal mixing; $\Delta S^{\text {mix }}=-R\left(X_{A} \ln X_{A}+X \ln X_{B}\right)$, where $X_{A}$ and $X_{B}$ are the molar fractions of components $A$ and $B$ in the mixture $\left(X_{A}+X_{B}=1\right)$. However, the enthalpy of mixing may be written as $\Delta \mathrm{H}^{\text {mix }}=\mathrm{X}_{\mathrm{A}} \mathrm{X}_{\mathrm{B}} \beta$, where $\beta$ is an interaction parameter lumping the energy of mixing contribution [3]. Under these assumptions, eq.(1) becomes:

$$
\Delta G^{m i x}=X_{A} X_{B} \beta+R T\left(X_{A} \ln X_{A}+X_{B} \ln X_{B}\right) .
$$

Figure 1. A phase diagram with a miscibility gap (lower frame) and a diagram of the free energy change (upper frame). The phase diagram is the temperature versus the molar fraction of a component e.g. $x_{B}$. Note that the diagram is symmetrical around $x_{B}=0.5$ which is the case of the regular solution model. Line (1) is the phase boundary. Above this line the two liquids are miscible and the system is stable (s). Below this line there is a metastable region $(\mathrm{m})$. Within that region the system is stable to small fluctuations but is unstable to large fluctuations. Line (2) is the spinodal. Below this line the system is unstable (u). Regions (m) and (u) constitute the miscibility gap. Within that gap the system turns from one phase to a two-phase system. Temperature $\left(T_{c}\right)$ is the upper consolute temperature. Above this temperature the two liquids are miscible in all proportions. At a given temperature $(\mathrm{T})$ the tie line (4) cuts the phase boundary and the spinodal at points $(a, c)$ and $(b, d)$, respectively. The

\footnotetext{
*E-mail address: favvas@chem.demokritos.gr ISSN: 1791-2377 @ 2008 Kavala Institute of Technology. All rights reserved.
}

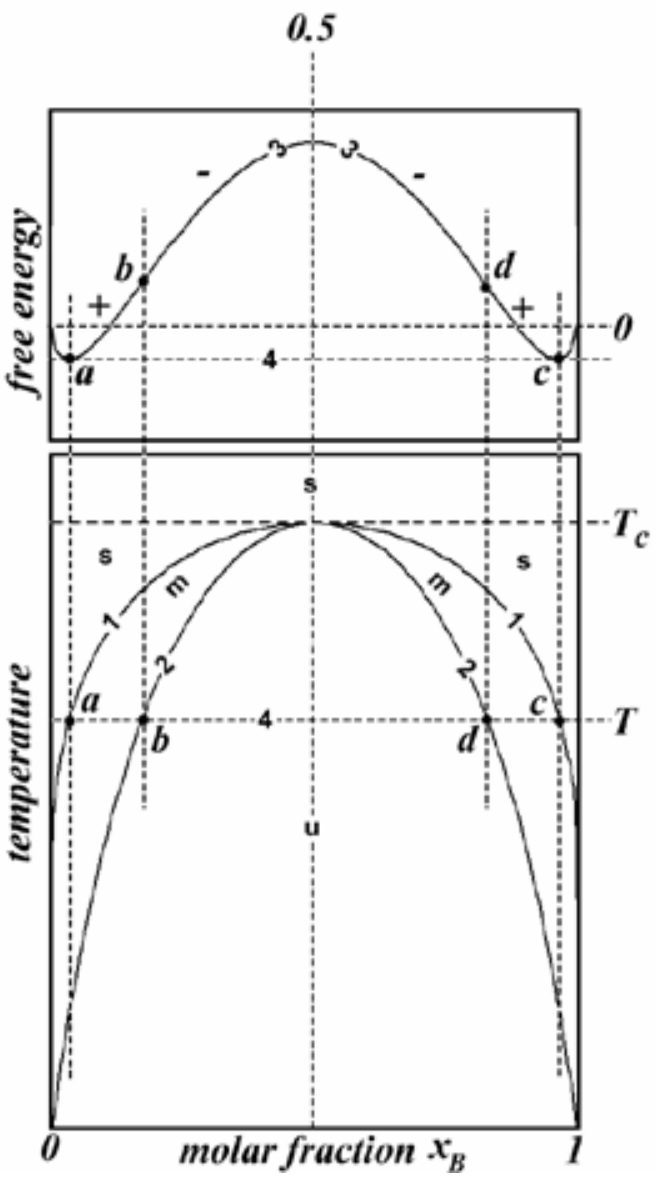

changes in the free energy of mixing $(\Delta G)$, at this given temperature, in respect to $\mathrm{x}_{\mathrm{B}}$ are shown by line (3) in the upper frame. Segments (ab) and (cd) correspond to a positive second derivative of $\Delta \mathrm{G}, \partial^{2} \Delta \mathrm{G} / \mathrm{x}_{\mathrm{B}}{ }^{2}>0$ while segment (bd) to a negative one, $\partial^{2} \Delta \mathrm{G} / \mathrm{x}_{\mathrm{B}}{ }^{2}<0$. At points (b) and (d) $\partial^{2} \Delta \mathrm{G} / \mathrm{x}_{\mathrm{B}}^{2}=0$. 


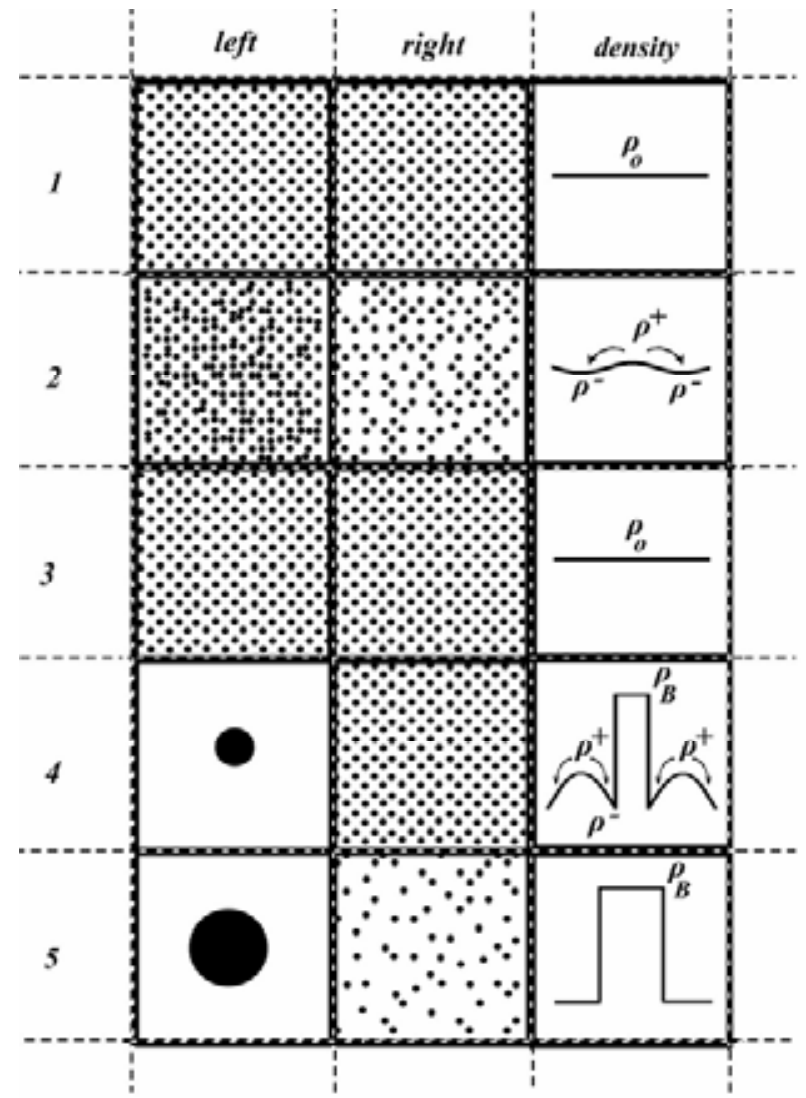

Figure 2 (left). The nucleation and growth process. The columns represent two adjacent local regions in the solution denoted as "left" and "right" and the rows five stages of the system denoted by numbers 1,2 , $3,4,5$; where the first three stages show what will happen when a small fluctuation occurs and the last two when a large fluctuation takes place. Black dots represent one of the two components e.g. B. In the $1^{\text {st }}$ row the solution has been brought in to the metastable region and at the moment there is not difference in the local concentrations; $C_{B}{ }^{\text {left }}=C_{B}{ }^{\text {right }}$ In the $2^{\text {nd }}$ row an infinitesimal fluctuation causes a concentration difference, such as $\mathrm{C}_{\mathrm{B}}{ }^{\text {left }}>\mathrm{C}_{\mathrm{B}}{ }^{\text {right }}$, and therefore diffusion is expected to take place between left and right localities. In the $3^{\text {rd }}$ row the expected diffusion results to $C_{B}{ }^{\text {left }}=C_{B}{ }^{\text {right }}$. Diffusion takes place from left to right i.e. from higher to lower concentration (down-hill) and the system comes back to what is depicted on the first row. In the $4^{\text {th }}$ row a large fluctuation causes the formation of a nucleus of a critical size. For presentation reasons, the figure was drawn such as all component $\mathrm{B}$ on the left region is spent to form that nucleus; therefore $\mathrm{C}_{\mathrm{B}}{ }^{\text {left }}=0<\mathrm{C}_{\mathrm{B}}{ }^{\text {right }}$. Down-hill diffusion takes place now from right to left; note, that the higher concentration is on the right, the nucleus does not intervene. Once some B reaches to the left is arrested by the nucleus that grows ( $5^{\text {th }}$ row). Phase separation occurring in this way is known as nucleation and growth and the fingerprint of that mechanism is the formation of a nucleus.

Figure 3 (right). The spinodal decomposition process. The columns denoted as "left" and "right" represent two adjacent local regions in the solution and the rows denoted by numbers " $1,2,3,4,5$," represent five stages of the system. Black dots represent one of the two components e.g. B. Note that rows 4 and 5 represent some final stages of the process.

The miscibility-gap and spinodal-region boundaries are obtained by calculating the first and second derivatives of the free energy, respectively, and setting them equal to zero.

$$
\frac{T}{T_{c}}=2 \frac{X_{A}-X_{B}}{\ln X_{A}-\ln X_{B}} ; \frac{T}{T_{c}}=4 X_{A} X_{B} ; T_{c}=\frac{\beta}{2 R}
$$

In Fig.1 (upper frame) it can be seen that the first derivative is zero at the two free energy minima corresponding to

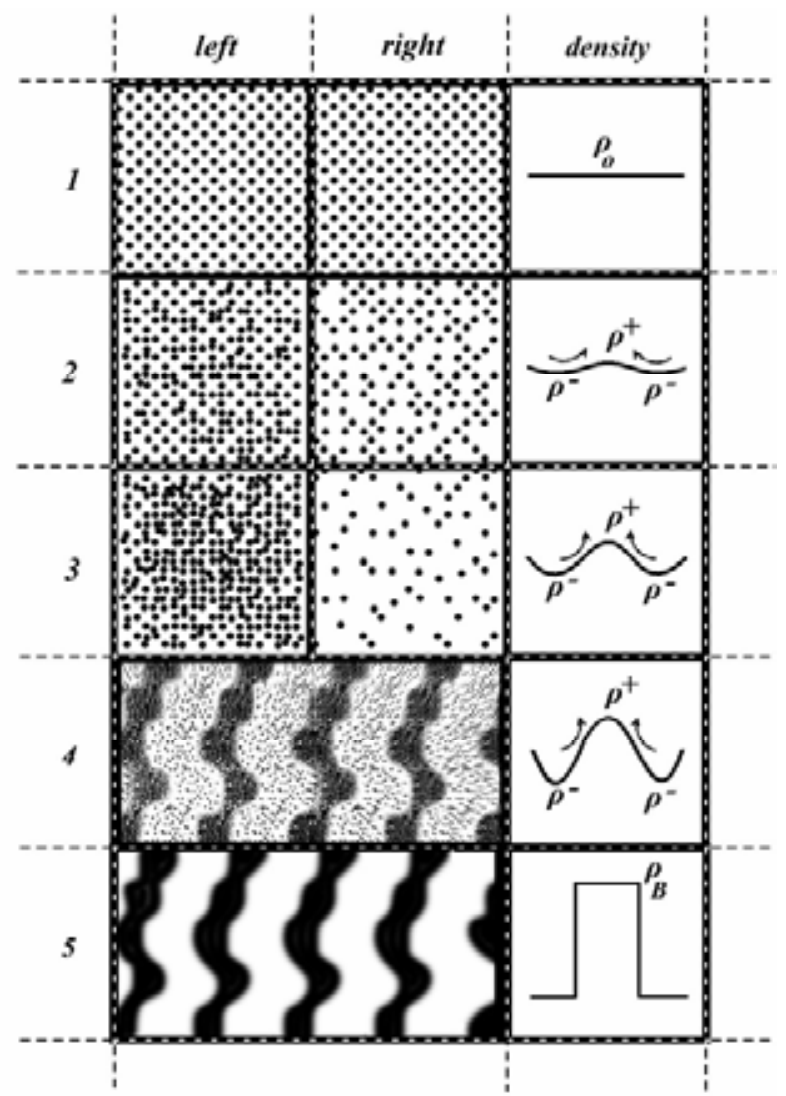

The drawing on the $4^{\text {th }}$ row is produced by repeating the $3^{\text {rd }}$ row to an $\mathrm{x}$ $y$ plane. The $5^{\text {th }}$ row is produced from the $4^{\text {th }}$ row by artwork. The column denoted as "density" represents the density profile of the system at a given stage. In the $1^{\text {st }}$ row the solution has been brought, very carefully, in to the unstable region. This carefulness is shown in a way that there is not difference in the local concentrations; $C_{B}{ }^{\text {left }}=C_{B}{ }_{B}$ right . Since there is not concentration gradient, no diffusion between left and right takes place; the solution remains at the moment one-phase system. The density of the system at this stage has a flat profile equal to an average value, let say $\rho_{\mathrm{o}}$. In the $2^{\text {nd }}$ row an infinitesimal fluctuation causes a concentration difference, such as $\mathrm{C}_{\mathrm{B}}{ }^{\text {left }}>\mathrm{C}_{\mathrm{B}}{ }^{\text {right }}$, and therefore diffusion is expected to take place between left and right localities. The density of the system has now a wavy profile with maxima and minima around the average density. In the third row the expected diffusion process results to $\mathrm{C}_{\mathrm{B}}{ }^{\text {left }}>>\mathrm{C}_{\mathrm{B}}{ }^{\text {right }}$, however. Diffusion does not take place from left to right i.e. from higher to lower concentration (downhill), but oppositely from right to left i.e. from lower to higher concentration (uphill). Note that if diffusion was taken place normally from left to right (downhill) it would result to $C_{B}{ }^{\text {left }}=C_{B}$ right; i.e. the system would be gone back to what is depicted on the first row. This can not be happened because is the case of a stable system; i.e. contradicts the initial statement that the system is already in the unstable region (compare also with Fig.2 to see the difference). The density is large in extent but small in degree; the arrows show the diffusion direction. As diffusion progresses the density increases until it reaches a point where becomes equal to the density of pure B (rows 3-5). Phase separation that occurs in this way is known as spinodal decomposition and the fingerprint of that mechanism is the uphill diffusion.

the two miscible phases and the second derivative is zero at the points of inflexion. Also, both derivatives are zero at the free energy maximum. For a series of temperatures, the locus of the free energy minima projected on a temperaturecomposition diagram defines the phase boundary and the locus of the points of inflexion the spinodal (see lower frame in Fig.1). The maxima coincide to the upper consolute temperature $T_{c}$ at $X_{B}=0.5$. Phase separation occurs when the system is within the miscibility gap. If the system is in the metastable region the mechanism is by nucleation and 
growth whereas if it is in the unstable region by spinodal decomposition.

Figure 2 depicts the nucleation and growth mechanism [4]. Gibbs (Ref.1 p.254) discussed the possibility of the formation of a fluid of different phase within any homogeneous fluid and showed that the work W required to form (by a reversible process) an heterogeneous globule in the interior of a very large mass having initially the uniform phase of the exterior mass will be:

$W=4 \pi r^{2} \gamma-\frac{4}{3} \pi r^{3} \Delta P$

where $\gamma$ is the surface tension, $r$ is the radius of the nucleus, and $\Delta \mathrm{P}$ is the hydrostatic pressure. In the case where a critical nucleus is formed the first derivative of eq.(4) becomes zero while the hydrostatic equilibrium is preserved; $\Delta \mathrm{P}=2 \gamma / \mathrm{r}_{\text {crit }}$, where $\mathrm{r}_{\text {crit }}$ is the radius of the critical nucleus. When $r>r_{\text {crit }}$ the nucleus grows and when $r<r_{\text {crit }}$ it collapses because the pressure exerted by the surface is greater than $\Delta \mathrm{P}$. By introducing $\mathrm{r}_{\text {crit }}$ in eq.(4) the minimum work $\mathrm{W}_{\min }$ required to form that critical nucleus is given as:

$$
W_{\min }=\frac{4}{3} \pi r_{c r i t}^{2} \gamma \text {. }
$$

Figure 3 depicts the spinodal decomposition mechanism [5]. Cahn and Hilliard [6] utilize the van der Waals equation for the energy of binary mixture [7] to develop a model for spinodal decomposition through Fick's law of diffusion.

$$
\frac{\partial C_{B}}{\partial t}=\left\{M\left(\frac{\partial f^{2}}{\partial C_{B}^{2}}\right)\right\}_{=D} \nabla^{2} C_{B}-2 M \kappa \nabla^{4} C_{B},
$$

where $f$ is the free energy density of homogeneous material of composition e.g. $\mathrm{C}_{\mathrm{B}}, \mathrm{M}$ is a positive constant [8], and $\kappa$ is a positive parameter. The first term on the right hand side of eq.(6) is the classical diffusion equation where the coefficient of $\nabla^{2} C_{B}$ is the diffusion coefficient $D$. Since $M>0$ the sign of $D$ will be determined by the sign of $\partial^{2} f / \partial C_{B}^{2}$. When $\partial^{2} f / \partial \mathrm{C}_{\mathrm{B}}^{2}>0$ the solution is stable, $\mathrm{D}>0$, and diffusion (if any) occurs downhill. When $\partial^{2} f / \partial \mathrm{C}_{\mathrm{B}}^{2}<0$ the solution has crossed the spinodal and is unstable, $\mathrm{D}<0$, and diffusion takes place uphill. It is noted, however, that the second term on the right hand side of eq.(6) (i.e. the fourth-order term) stabilizes the system against short distance scale fluctuations when $\partial^{2} f / \partial C_{B}^{2}<0$. Uphill diffusion and consequently spinodal decomposition requires a large enough distance scale fluctuation (i.e. to move mass over long distances).

In this note an elementary review on the concept of spinodal decomposition was given. The nucleation and growth mechanism was also presented. Comparing the two processes it is evident that nucleation is large in degree and small in extent while spinodal is small in degree and large in extent. In Fig. 2 and 3 the evolution of the density profiles at various stages of the processes was drawn. It is noted that spinodal decomposition results to a high interconnectivity of the two phases. However, the morphology of the resulted separation is only an indication that spinodal decomposition has taken place [9]. Nucleation may also produce high interconnectivity [10]. Besides, fractal morphology is possible to be induced by both processes. For instance a fractal flake that grows is an example of a fractally nucleation process whereas a twin-dragon Peano curve that thickens is an example of a fractally spinodal process [11].

Acknowledgments: The authors would like to thank Archimedes research Project and INTERREG-III 'Hybrid Technology for separation' for funding this work.

\section{References}

1. J.W.Gibbs, Collected Works, Yale University Press, New Haven, pp.105-115 and pp.252-258 (1948).

2. E.A.Guggenheim, Proc.Roy.Soc.A 148, 304 (1935); J.B.Thompson, Jr., in Researches in Geochemistry, Ed. by P.H.Abelson. John Wiley and Sons Inc., New York, Vol.2 p.340 (1967).

3. For the ideal solution the enthalpy of mixing is zero.

4. IUPAC definition of nucleation and growth: A process in a phase transition in which nuclei of a new phase are first formed, followed by the propagation of the new phase at a faster rate; J.B.Clark, J.W.Hastie, L.H.E.Kihlborg, R.Metselaar, and M.M.Thackeray, Pure App.Chem. 66, 577 (1994).

5. IUPAC definition of spinodal decomposition: A clustering reaction in a homogeneous, supersaturated solution (solid or liquid) which is unstable against infinitesimal fluctuations in density or composition. The solution therefore separates spontaneously into two phases, starting with small fluctuations and proceeding with a decrease in the Gibbs energy without a nucleation barrier; ibid.

6. J.W.Chan and J.E.Hilliard, J.Chem.Phys. 28, 258 (1958).; J.W.Chan and J.E.Hilliard, J.Chem.Phys. 31, 688 (1959); see also J.W.Chan, J.Chem.Phys. 30, 1121 (1959); R.B.Heady and J.W.Chan, J.Chem.Phys. 58, 896 (1973); J.W.Cahn, Trans.A.IM.E. 242, 166 (1968); J.W.Cahn, Acta Met. 9, 795 (1961); J.W.Cahn and R.J.Charles, Phys.Chem. Glasses 6, 181 (1965); V.S.Stubican and A.H.Schultz, J. Amer. Cerum. Soc., 51290 (1968); A.Noviek-Cohen,
J.Stat.Phys. 38, 707 (1985); V.M.Agishev and K.M.Yamaleev, Iz.Vyss.U.Zav. Fizika 10, 124 (1975); K.R.Mecke and V.Sofonea, Phys.Rev.E 56, R3761 (1997); J.Zhu, L-Q.Chen, J.Shen, and V.Tikare, Phys.Rev.E 60, 3564 (1999); C.P.Grant, Commun.Part.Differ.Eq. 18, 453 (1993).

7. J.D.van der Waals, The thermodynamic theory of capillarity flow under the hypothesis of a continuous variation in density, Verhandelingen der Koninklijke Nederlandsche Akademie van Wetenschappen te Amsterdam 1, 1 (1893).

8. $\mathrm{M}$ is related to the interdiffusion coefficient. For a binary system $\mathrm{J}_{\mathrm{B}}=-\mathrm{M} \cdot \nabla\left(\mu_{\mathrm{B}}-\mu_{\mathrm{A}}\right)$, where $\mathrm{J}$ and $\mu$ are respectively the flux and the chemical potential of e.g. the B component and $\mathrm{M}$ is the mechanical mobility. Changes in the concentration are given by taking the divergence of the flux: $\partial \mathrm{C}_{\mathrm{B}} / \partial \mathrm{t}=-\nabla \cdot \mathrm{J}_{\mathrm{B}}$

9. J.W.Cahn, J.Chem.Phys. 42, 93 (1965)

10. W.Haller, J.Chem.Phys. 42, 686 (1965); see also W.Haller, D.H.Blackburn, J.H.Simmons, F.E.Wagstaff, and R.J.Charles, J. Amer. Ceram. Soc. 53, 34 (1970); G.R.Srinivasan, I.Tweer, P.B.Macedo, A.Sarkar, and W.Haller, J.Non-Cryst. Solids 6, 221 (1971); W.Haller, D.H.Blackburn, and J.H.Simmons, J. Amer. Cerum. Soc., 57126 (1974).

11. F.Katsaros, P.Makri. A.Ch.Mitropoulos, N.Kanellopoulos, U.Keiderling, and A.Wiedenmann, Physica B 234-236, 402 (1997). 